\title{
Reflection implies the SCH
}

\author{
by \\ Saharon Shelah (Jerusalem and New Brunswick, NJ)
}

\begin{abstract}
We prove that, e.g., if $\mu>\operatorname{cf}(\mu)=\aleph_{0}$ and $\mu>2^{\aleph_{0}}$ and every stationary family of countable subsets of $\mu^{+}$reflects in some subset of $\mu^{+}$of cardinality $\aleph_{1}$, then the SCH for $\mu^{+}$holds (moreover, for $\mu^{+}$, any scale for $\mu^{+}$has a bad stationary set of cofinality $\aleph_{1}$ ). This answers a question of Foreman and Todorčević who get such a conclusion from the simultaneous reflection of four stationary sets.
\end{abstract}

0. Introduction. In $\S 1$ we prove that the strong hypothesis $\left(\operatorname{pp}(\mu)=\mu^{+}\right.$ for every singular $\mu$ ) and hence the SCH (singular cardinal hypothesis, that is, $\lambda^{\kappa} \leq \lambda^{+}+2^{\kappa}$ ) holds when for every $\lambda \geq \aleph_{1}$ every stationary $\mathscr{S} \subseteq[\lambda]^{\aleph_{0}}$ reflects in some $A \in[\lambda]^{\aleph_{1}}$.

This answers a question of Foreman and Todorčević [FoTo] who proved that the SCH holds for every $\lambda \geq \aleph_{1}$ when any four stationary $\mathscr{S}_{l} \subseteq[\lambda]^{\aleph_{0}}$, $l=1,2,3,4$, reflect simultaneously in some $A \in[\lambda]^{\aleph_{1}}$. They were probably motivated by Veličković [Ve92a] who used another reflection principle: for every stationary $\mathscr{A} \subseteq[\lambda]^{\aleph_{0}}$ there is $A \in[\lambda]^{\aleph_{1}}$ such that $\mathscr{A} \cap[A]^{\aleph_{0}}$ contains a closed unbounded subset, rather than just a stationary set.

The proof here is self-contained modulo two basic quotations from [Sh:g], [Sh:f]; we continue [Sh:e], [Sh 755] in some respects. We prove more in $\S 1$. In particular if $\mu>\operatorname{cf}(\mu)=\aleph_{0}$ and $\operatorname{pp}(\mu)>\mu^{+}$then some $\mathscr{A} \subseteq\left[\mu^{+}\right]^{\aleph_{0}}$ reflect in no uncountable $A \in\left[\mu^{+}\right] \leq \mu$ (see more at the end).

We thank the referee and Shimoni Garti for quite a few helpful comments.

For the reader's convenience let us recall some basic definitions.

2000 Mathematics Subject Classification: 03E04, 03E05.

Key words and phrases: reflection, stationary sets, Singular Cardinal Hypothesis, pcf, set theory.

The author would like to thank the Israel Science Foundation for partial support of this research (Grant no. 242/03). Publication 794.

I would like to thank Alice Leonhardt for the beautiful typing. 
0.1. Definition. Assume $\theta$ is regular uncountable (if $\theta=\sigma^{+},[B]^{<\sigma}=$ $[B] \leq \theta$, we can use $[B]^{\leq \theta}$; the main case is $B=\lambda$ ).

(a) $\mathscr{A} \subseteq[B]^{<\theta}$ is closed in $[B]^{<\theta}$ if for every $\left\{x_{\beta}: \beta<\alpha\right\} \subseteq \mathscr{A}$ where $0<\alpha<\theta$ and $\beta_{1}<\beta_{2}<\alpha \Rightarrow x_{\beta_{1}} \subseteq x_{\beta_{2}}$, we have $\bigcup_{\beta<\alpha} x_{\beta} \in \mathscr{A}$.

(b) $\mathscr{A}$ is unbounded in $[B]^{<\theta}$ if for any $y \in[B]^{<\theta}$ we can find $x \in \mathscr{A}$ such that $x \supseteq y$.

(c) $\mathscr{A}$ is a club in $[B]^{<\theta}$ if $\mathscr{A} \subseteq[B]^{<\theta}$ and (a)+(b) hold for $\mathscr{A}$.

(d) $\mathscr{A}$ is stationary in $[B]^{<\theta}$, or is a stationary subset of $[B]^{<\theta}$, if $\mathscr{A} \subseteq$ $[B]^{<\theta}$ and $\mathscr{A} \cap \mathscr{C} \neq \emptyset$ for every club $\mathscr{C}$ of $[B]^{<\theta}$.

(e) Similarly for $[B]^{\leq \theta}$ or $[B]^{\theta}$ or consider $\mathscr{S} \subseteq[B]^{\leq \theta}$ as a subset of $[B]^{<\theta^{+}}$.

0.2. Remark. If $B=\theta$ then $\mathscr{A} \subseteq[B]^{<\theta}$ is stationary iff $\mathscr{A} \cap \theta$ is a stationary subset of $\theta$.

0.3. Definition. Let $\mathscr{A} \subseteq\left[B_{1}\right]^{<\theta}$ and $B_{2} \in\left[B_{1}\right]^{\mu}$. We say that $\mathscr{A}$ reflects in $B_{2}$ when $\mathscr{A} \cap\left[B_{2}\right]^{<\theta}$ is a stationary subset of $\left[B_{2}\right]^{<\theta}$.

0.4. Definition. Let $\kappa$ be a regular uncountable cardinal, and assume $\mathscr{A}$ is a stationary subset of $[B]^{<\kappa}$. We define $\nabla_{\mathscr{A}}$ (i.e., the diamond principle for $\mathscr{A})$ to be the following assertion: there exists a sequence $\left\langle u_{a}: a \in \mathscr{A}\right\rangle$ such that $u_{a} \subseteq a$ for any $a \in \mathscr{A}$, and for every $B^{\prime} \subseteq B$ the set $\{a \in \mathscr{A}$ : $\left.B^{\prime} \cap a=u_{a}\right\}$ is stationary in $\left[B^{\prime}\right]^{<\kappa}$.

0.5. Notation.

(1) For regular $\lambda>\kappa$ let $S_{\kappa}^{\lambda}=\{\delta<\lambda: \operatorname{cf}(\delta)=\kappa\}$.

(2) $\mathscr{H}(\lambda)$ is the set of $x$ with transitive closure of cardinality $<\lambda$.

(3) $<_{\lambda}^{*}$ denotes any well ordering of $\mathscr{H}(\lambda)$.

Let us recall the definition of the next ideal (see [Sh:E12]):

0.6. Definition. For $S \subseteq \lambda$ we say that $S \in \check{I}[\lambda]$ if there is a club $E$ in $\lambda$ and a sequence $\left\langle C_{\alpha}: \alpha<\lambda\right\rangle$ such that:

(i) $C_{\alpha} \subseteq \alpha$ for every $\alpha<\lambda$,

(ii) $\operatorname{otp}\left(C_{\alpha}\right)<\alpha$,

(iii) $\beta \in C_{\alpha} \Rightarrow C_{\beta}=\beta \cap C_{\alpha}$,

(iv) $\alpha \in E \cap S \Rightarrow \alpha=\sup \left(C_{\alpha}\right)$.

0.7. Claim (by [Sh 420] or see [Sh:E12]).

(1) If $\kappa, \lambda$ are regular and $\lambda>\kappa^{+}$, then there is a stationary $S \subseteq S_{\kappa}^{\lambda}$ such that $S \in \check{I}[\lambda]$.

(2) In 0.6 we can add $\alpha \in E \cap S \Rightarrow \operatorname{otp}\left(C_{\alpha}\right)=\operatorname{cf}(\alpha)$.

0.8. Definition/Observation. Let $\mathscr{A} \subseteq[\lambda]^{\theta}$ be stationary and $\lambda \geq$ $\sigma>\theta$ and suppose $\sigma$ has uncountable cofinality. $\operatorname{Set}_{\operatorname{prj}}(\mathscr{A}):=\{\sup (a \cap \sigma)$ : $a \in \mathscr{A}\}$. It is a stationary subset of $\sigma$; if $\sigma=\lambda$ we may omit it in notation. 
0.9. Definition. Let $I$ be a set of ordinals and $f_{i}$ be a function with domain $\aleph_{0}$ to the ordinals, for every $i \in I$. We say that the sequence $\bar{f}=$ $\left\langle f_{i}: i \in I\right\rangle$ is free if we can find a sequence $\bar{n}=\left\langle n_{i}: i \in I\right\rangle$ of natural numbers such that $\left(i, j \in I \wedge i<j \wedge n_{i}, n_{j} \leq n<\omega\right) \Rightarrow f_{i}(n)<f_{j}(n)$. We say that $\bar{f}$ is $\mu$-free when for every $J \in[I]^{<\mu}$ the sequence $\bar{f}\lceil J$ is free.

0.10. Remark. If we consider " $\left\langle f_{\alpha}: \alpha \in S\right\rangle$ for some stationary $S \subseteq \theta$ " when $\theta=\operatorname{cf}(\theta)>\aleph_{0}$, then we can assume (without loss of generality) that $n_{i}=n(*)$ for every $i \in S$, as we can decrease $S$.

\section{Reflection in $\left[\mu^{+}\right]^{\aleph_{0}}$ and the strong hypothesis}

\subsection{The Main Claim. Assume}

(A) $\lambda=\mu^{+}$and $\mu>\operatorname{cf}(\mu)=\aleph_{0}$ and $\aleph_{2} \leq \mu_{*} \leq \lambda$ (e.g., $\mu_{*}=\aleph_{2}$, which implies that below always $\theta=\aleph_{1}$ ).

(B) $\bar{\lambda}=\left\langle\lambda_{n}: n<\omega\right\rangle$ is an increasing sequence of regular cardinals $>\aleph_{1}$ with limit $\mu$ and $\lambda=\operatorname{tcf}\left(\prod_{n<\omega} \lambda_{n},<_{J_{\omega}}\right)$.

(C) $\bar{f}=\left\langle f_{\alpha}: \alpha<\lambda\right\rangle$ is $<_{J_{\omega}^{\text {bd }}}$-increasing cofinal in $\left(\prod_{n<\omega} \lambda_{n},<_{J_{\omega}^{\text {bd }}}\right)$.

(D) The sequence $\bar{f}$ is $\mu_{*}$-free or at least for every cardinal $\theta$ for which $\aleph_{1} \leq \theta=\operatorname{cf}(\theta)<\mu_{*}$ the following is satisfied: if $\theta \leq \sigma<\mu_{*}$ and $\mathscr{A} \subseteq[\sigma]^{\aleph_{0}}$ is stationary (recall 0.2 ), and $\left\langle\delta_{i}: i<\theta\right\rangle$ is an increasing continuous sequence of ordinals $<\lambda$, then for some stationary subfamily $\mathscr{A}_{1}$ of $\mathscr{A}\left(\mathscr{A}_{1}\right.$ is stationary in $[\sigma]^{\aleph_{0}}$ of course), if we let $R_{1}=\operatorname{prj}_{\theta}\left(\mathscr{A}_{1}\right)$ (see 0.8), then $\left\langle f_{\delta_{i}}: i \in R_{1}\right\rangle$ is free (see 0.9). By 0.10 we can assume that $i \in R_{1} \Rightarrow n_{i}=n(*)$ so $\left\langle f_{\delta_{i}}(n): i \in R_{1}\right\rangle$ is strictly increasing for every $n \in[n(*), \omega)$.

Then some stationary $\mathscr{A} \subseteq[\lambda]^{\aleph_{0}}$ does not reflect in any $A \in[\lambda]^{\aleph_{1}}$ or even in any uncountable $A \in[\lambda]^{<\mu_{*}}$ (see Definition 0.3 ).

1.2. Remark. (0) From the main claim the result on SCH should be clear from pcf theory (by translating between the pp, cov and cardinal arithmetic) but we shall give details (i.e. quotes).

(1) Clause (D) from Claim 1.1 is related to "the good set of $\bar{f}, \operatorname{gd}(\bar{f})$, contains $S_{\theta}^{\lambda}$ modulo the club filter". But (D) is stronger.

Note that $\operatorname{gd}(\bar{f})=\left\{\delta<\lambda: \aleph_{0}<\operatorname{cf}(\delta)<\mu\right.$ and for some increasing sequence $\left\langle\alpha_{i}: i<\operatorname{cf}(\delta)\right\rangle$ of ordinals with limit $\delta$ and a sequence $\bar{n}=\left\langle n_{i}\right.$ : $i<\operatorname{cf}(\delta)\rangle$ of natural numbers we have $\left(i<j<\operatorname{cf}(\delta) \wedge n_{i} \leq n<\omega \wedge n_{j} \leq n\right.$ $\left.<\omega) \Rightarrow f_{\alpha_{i}}(n)<f_{\alpha_{j}}(n)\right\}$ (so $\left\langle\bigcup\left\{f_{\alpha_{i}}(n): i<\operatorname{cf}(\delta)\right.\right.$ and $\left.\left.n \geq n_{i}\right\}: n<\omega\right\rangle$ is a $<_{J_{\omega}^{\text {bd }}}-$ eub of $\bar{f}\lceil\delta$ ).

If we use another ideal, $J$ say, on $\delta<\mu$, then $n_{i}$ is replaced by $s_{i} \in J$. 
(2) Recall that by using the silly square ([Sh:g, II, 1.5A, p. 51]), if $\operatorname{cf}(\mu)$ $\leq \theta<\mu, J$ is an ideal on $\theta$ (e.g. $\theta=\aleph_{0}, J=J_{\omega}^{\text {bd }}$ ) and $\operatorname{pp}_{J}(\mu)>\lambda=$ $\operatorname{cf}(\lambda)>\mu$, then we can find a sequence $\left\langle\lambda_{i}: i<\theta\right\rangle$ of regulars $<\mu$ such that $\mu=\lim _{J}\left\langle\lambda_{i}: i<\theta\right\rangle$ and $\operatorname{tcf}\left(\prod_{i<\theta} \lambda_{i},<_{J}\right)=\lambda$, and some $\bar{f}=\left\langle f_{\alpha}: \alpha<\lambda\right\rangle$ exemplifying it satisfies $\operatorname{gd}(\bar{f})=\{\delta<\lambda: \theta<\operatorname{cf}(\delta)<\mu\}$; moreover $\bar{f}$ is $\mu^{+}$-free, which here means that for every $u \subseteq \lambda$ of cardinality $\leq \mu$ we can find $\left\langle s_{\alpha}: \alpha \in u\right\rangle$ such that $s_{\alpha} \in J$, and for $\alpha<\beta$ from $u$ we have $\varepsilon \in \theta \backslash\left(s_{\alpha} \cup s_{\beta}\right) \Rightarrow f_{\alpha}(\varepsilon)<f_{\beta}(\varepsilon)$. This is stronger than the demand in clause (D).

(3) Also recall that if $\kappa$ is supercompact, $\mu>\kappa>\theta=\operatorname{cf}(\mu)$ and $\left\langle\lambda_{i}\right.$ : $i<\theta\rangle$ is an increasing sequence of regulars with limit $\mu$, and $\left\langle f_{\alpha}: \alpha<\lambda\right\rangle$ exemplifies $\lambda=\mu^{+}=\operatorname{tcf}\left(\prod_{i<\theta} \lambda_{i},<_{J_{\theta}}\right.$ bd $)$, then for unboundedly many $\kappa^{\prime} \in$ $\kappa \cap \operatorname{Reg} \backslash \theta^{+}$the set $S_{\kappa^{\prime}}^{\lambda} \backslash \operatorname{gd}(\bar{f})$ is stationary. This is preserved by e.g. $\operatorname{Levy}\left(\aleph_{1},<\kappa\right)$.

(4) For part of the proof (mainly Subclaim 1.5) we can weaken clause (D) of the assumption, e.g. at the end demand " $\Rightarrow f_{\delta_{i}}(n) \neq f_{\delta_{j}}(n)$ " only. The weakest version of clause (D) which suffices there is: for any club $C$ of $\theta$ the set $\bigcup\left\{\operatorname{Rang}\left(f_{\alpha}\right): \alpha \in C\right\}$ has cardinality $\theta$.

Before proving 1.1 we draw some conclusions.

\subsection{Conclusion.}

(1) Assume $\mu>2^{\aleph_{0}}$. Then $\mu^{\aleph_{0}}=\mu^{+}$provided that

$(\mathrm{A})_{\mu} \mu>\operatorname{cf}(\mu)=\aleph_{0}$,

(B) $\mu_{\mu}$ every stationary $\mathscr{A} \subseteq\left[\mu^{+}\right]^{\aleph_{0}}$ reflects in some $A \in\left[\mu^{+}\right]^{\aleph_{1}}$.

(2) Assume $\mu \geq \mu_{*} \geq \aleph_{2}$. We can replace $(\mathrm{B})_{\mu}$ by

(B) $\mu_{\mu_{*}}$ every stationary $\mathscr{A} \subseteq\left[\mu^{+}\right]^{\aleph_{0}}$ reflects in some uncountable $A \in\left[\mu^{+}\right]^{<\mu_{*}}$.

Proof. (1) Clearly if $\aleph_{1} \leq \mu^{\prime} \leq \mu$ then (B) $\mu_{\mu^{\prime}}$ holds. Now if $\mu$ is a counterexample, without loss of generality it is a minimal counterexample, and then by [Sh:g, IX, §1] we have $\operatorname{pp}(\mu)>\mu^{+}$; hence there is a sequence $\left\langle\lambda_{n}^{0}\right.$ : $n<\omega\rangle$ of regular cardinals with limit $\mu$ such that $\mu^{++}=\operatorname{tcf}\left(\prod_{n<\omega} \lambda_{n}^{0} / J_{\omega}^{\text {bd }}\right)$ (see [Sh:g]; more in [Sh:E12] or [Sh 430, 6.5]; e.g. using "no hole for pp" and the pcf theorem). Let $\bar{f}^{0}=\left\langle f_{\alpha}^{0}: \alpha<\mu^{++}\right\rangle$witness this. Hence by [Sh:g, II, $1.5 \mathrm{~A}$, p. 51] there is an $\bar{f}$ as required in 1.1 , even a $\mu^{+}$-free one, and also the other assumptions there hold, so we can conclude that there exists $\mathscr{A} \subseteq\left[\mu^{+}\right]^{\aleph_{0}}$ which does not reflect in any $A \in\left[\mu^{+}\right]^{\aleph_{1}}$, getting a contradiction to $(\mathrm{B})_{\mu}$.

(2) The same proof. 


\subsection{Conclusion.}

(1) If for every $\lambda>\aleph_{1}$, every stationary $\mathscr{A} \subseteq[\lambda]^{\aleph_{0}}$ reflects in some $A \in[\lambda]^{\aleph_{1}}$, then

(a) the strong hypothesis (see [Sh 410], [Sh 420], [Sh:E12]) holds, i.e. for every singular $\mu, \operatorname{pp}(\mu)=\mu^{+}$, and moreover $\operatorname{cf}\left([\mu]^{\operatorname{cf}(\mu)}, \subseteq\right)$ $=\mu^{+}$(which follows),

(b) the SCH holds.

(2) Let $\theta \geq \aleph_{0}$. We can restrict ourselves to $\lambda>\theta^{+}$and $A \in[\lambda]^{\theta^{+}}$ (getting the strong hypothesis and $S C H$ above $\theta$ ).

Proof. (1) As in 1.3, by 1.1 we have $\mu>\operatorname{cf}(\mu)=\aleph_{0} \Rightarrow \operatorname{pp}(\mu)=\mu^{+}$, which implies clause (a) (i.e. by [Sh:g, VIII, §1], $\mu>\operatorname{cf}(\mu) \Rightarrow \operatorname{pp}(\mu)=\mu^{+}$). Hence inductively by [Sh:g, IX, 1.8, p. 369], [Sh 430, 1.1] we have $\kappa<\mu \Rightarrow$ $\operatorname{cf}\left([\mu]^{\kappa}, \subseteq\right)$ is $\mu$ if $\operatorname{cf}(\mu)>\kappa$ and is $\mu^{+}$if $\mu>\kappa \geq \operatorname{cf}(\mu)$. This is a consequence of the strong hypothesis. The SCH follows.

(2) The same proof. $\mathbf{m}_{1.4}$

Proof of 1.1. Let $M^{*}$ be an algebra with universe $\lambda$ and countably many functions, e.g. all those definable in $\left(\mathscr{H}\left(\lambda^{+}\right), \in,<_{\lambda^{+}}^{*}, \bar{f}\right)$ and mapping $\lambda$ to $\lambda$ or just the functions $\alpha \mapsto f_{\alpha}(n), \alpha \mapsto \alpha+1$.

1.5. Subclaim. There are $\bar{S}, S^{*}, \bar{D}$ such that:

$(*)_{1} \bar{S}=\left\langle S_{\varepsilon}: \varepsilon<\omega_{1}\right\rangle$ is a sequence of pairwise disjoint stationary subsets of $S_{\aleph_{0}}^{\lambda}$,

$(*)_{2}$ (i) $S^{*} \subseteq S_{\aleph_{1}}^{\lambda}=\left\{\delta<\lambda: \operatorname{cf}(\delta)=\aleph_{1}\right\}$ is stationary and belongs to $\check{I}[\lambda]$

(ii) if $\delta \in S^{*}$ then there is an increasing continuous sequence $\left\langle\alpha_{\varepsilon}\right.$ : $\left.\varepsilon<\omega_{1}\right\rangle$ of ordinals with limit $\delta$ such that for some sequence $\bar{\zeta}=\left\langle\zeta_{\varepsilon}: \varepsilon \in R\right\rangle$ of ordinals $<\omega_{1}$, the set $R \subseteq \omega_{1}$ is stationary, $\varepsilon \in R \Rightarrow \alpha_{\varepsilon} \in S_{\zeta_{\varepsilon}}$, and $\bar{\zeta}$ is with no repetitions,

$(*)_{3}$ (i) $\bar{D}=\left\langle\left(D_{1, \varepsilon}, D_{2, \varepsilon}\right): \varepsilon<\omega_{1}\right\rangle$,

(ii) $D_{l, \varepsilon}$ is a filter on $\omega$ containing the filter of cobounded subsets of $\omega$,

(iii) if $R_{1} \subseteq \omega_{1}$ is unbounded and $A \in \bigcap\left\{D_{1, \varepsilon}: \varepsilon \in R_{1}\right\}$ then for some $\varepsilon \in R_{1}$ we have $A \neq \emptyset \bmod D_{2, \varepsilon}$,

(iv) for each $\varepsilon<\omega_{1}$ for some $A$ we have $A \in D_{1, \varepsilon} \& \omega \backslash A \in D_{2, \varepsilon}$.

1.6. Remark. (1) For 1.5 we can assume (A), (B), (C) of 1.1 and weaken clause (D): because (in the proof below) necessarily for any stationary $S^{*} \subseteq$ $S_{\aleph_{1}}^{\lambda}$ which belongs to $\check{I}[\lambda]$, we can restrict the demand in (D) of 1.1 for any $\left\langle\delta_{i}: i<\theta\right\rangle$ with limit in $S^{*}$. See more in [Sh 775]. 
(2) In Subclaim 1.5 we can demand $\zeta_{\varepsilon}=\varepsilon$ in $(*)_{2}($ ii). See the proof.

(3) If we like to demand that each $D_{l, \varepsilon}$ is an ultrafilter (or just have " $A \in D_{2, \varepsilon}$ " in the end of $(*)_{3}($ iii) of 1.5$)$, use [Sh:E3].

Proof of Subclaim 1.5. How do we choose $\bar{S}, S^{*}, \bar{D}$ ?

Let $\left\langle A_{i}: i \leq \omega_{1}\right\rangle$ be a sequence of infinite pairwise almost disjoint subsets of $\omega$. Let $D_{1, i}=\left\{A \subseteq \omega: A_{i} \backslash A\right.$ is finite $\}$ and $D_{2, i}=\left\{A \subseteq \omega: A_{j} \backslash A\right.$ is finite for all but finitely many $\left.j<\omega_{1}\right\}$, so $D_{2, i}$ does not depend on $i$. Clearly $\left\langle\left(D_{1, i}, D_{2, i}\right): i<\omega_{1}\right\rangle$ satisfies $(*)_{3}$.

Recall that by 0.7 and the fact that $\lambda>\aleph_{\omega}>\aleph_{2}$, there is a stationary $S^{*} \subseteq S_{\aleph_{1}}^{\lambda}$ from $\check{I}[\lambda]$, and so every stationary $S^{\prime} \subseteq S^{*}$ has the same properties (i.e. is a stationary subset of $\lambda$ which belongs to $\check{I}[\lambda]$ and is included in $S_{\aleph_{1}}^{\lambda}$ ).

Let $N \prec\left(\mathscr{H}\left(\left(2^{\lambda}\right)^{+}\right), \in,<^{*}\right)$ be of cardinality $\mu$ such that $\mu+1 \subseteq N$ and $\{\bar{\lambda}, \mu, \bar{f}\}$ belongs to $N$. Let $C^{*}=\bigcap\{C: C \in N$ is a club of $\lambda\}$, so clearly $C^{*}$ is a club of $\lambda$. For each $h \in{ }^{\lambda}\left(\omega_{1}\right)$ we can try $\bar{S}^{h}=\left\langle S_{\gamma}^{h}: \gamma<\omega_{1}\right\rangle$ where $S_{\gamma}^{h}=\left\{\delta<\lambda: \operatorname{cf}(\delta)=\aleph_{0}\right.$ and $\left.h(\delta)=\gamma\right\}$, so it is enough to show that for some $h \in N$, the sequence $\bar{S}^{h}$ is as required. As $\|N\|<\lambda$, for this it is enough to show that for every $\delta \in S_{\aleph_{1}}^{\lambda} \cap C^{*}$ (or just for every $\delta \in S^{*} \cap C^{*}$, or just for stationarily many $\left.\delta \in S^{*} \cap C^{*}\right)$ the demand holds for $\bar{S}^{h}$ for some $h \in\left({ }^{\lambda}\left(\omega_{1}\right)\right) \cap N$. That is, $S^{\bar{h}}$ satisfies $(*)_{1}$ and $(*)_{2}($ ii) of Subclaim 1.5. Given any $\delta \in S_{\aleph_{1}}^{\lambda} \cap C^{*}$ let $\left\langle\alpha_{\varepsilon}: \varepsilon<\omega_{1}\right\rangle$ be an increasing continuous sequence of ordinals with limit $\delta$, without loss of generality $\varepsilon<\omega_{1} \Rightarrow \operatorname{cf}\left(\alpha_{\varepsilon}\right)=\aleph_{0}$, and by assumption (D) of 1.1 for some $\left({ }^{1}\right)$ stationary $R \subseteq \omega_{1}$ and $n=n(*)<\omega$, the sequence $\left\langle f_{\alpha_{\varepsilon}}(n): \varepsilon \in R\right\rangle$ is strictly increasing; let its limit be $\beta^{*}$. So $\beta^{*} \leq \mu$ and $\operatorname{cf}\left(\beta^{*}\right)=\aleph_{1}$ but $\mu+1 \subseteq N$, hence $\beta^{*} \in N$.

Note that

$\otimes_{1}$ for every $\beta^{\prime}<\beta^{*}$ the set $\left\{\alpha \in S_{\aleph_{0}}^{\lambda}: f_{\alpha}(n(*)) \in\left[\beta^{\prime}, \beta^{*}\right)\right\}$ is a stationary subset of $\lambda$.

[Why? Assume that $\beta^{\prime}<\beta^{*}$ and that the set $S^{\prime}=\left\{\alpha \in S_{\aleph_{0}}^{\lambda}: f_{\alpha}(n(*)) \in\right.$ $\left.\left[\beta^{\prime}, \beta^{*}\right)\right\}$ is not a stationary subset of $\lambda$. As $\beta^{*}+1 \subseteq N$ and $\bar{f} \in N$, clearly $S^{\prime} \in N$, hence there is a club $C^{\prime}$ of $\lambda$ disjoint from $S^{\prime}$ which belongs to $N$. Clearly $\operatorname{acc}\left(C^{\prime}\right)$ too is a club of $\lambda$ which belongs to $N$, hence $C^{*} \subseteq \operatorname{acc}\left(C^{\prime}\right)$, hence $\delta \in \operatorname{acc}\left(C^{\prime}\right)$. So $\delta=\sup \left(C^{\prime} \cap \delta\right)$, so $C^{\prime} \cap \delta$ is a club of $\delta$. Recall that $\left\{\alpha_{\varepsilon}: \varepsilon \in R\right\}$ is a stationary subset of $\delta$ of order type $\aleph_{1}$.

Now by the choice of $\beta^{*}$, for some $\varepsilon(*) \in R$ we have $\beta^{\prime} \leq f_{\alpha_{\varepsilon(*)}}(n(*))$, hence $\varepsilon \in R \backslash \varepsilon(*) \Rightarrow f_{\alpha_{\varepsilon}}(n(*)) \in\left[\beta^{\prime}, \beta^{*}\right)$, so $\delta$ has a stationary subset included in $S^{\prime}$, hence disjoint from $C^{\prime}$, a contradiction.]

$\left({ }^{1}\right)$ Note that if we just require that $\left\langle f_{\alpha_{\varepsilon}}(n): \varepsilon \in R\right\rangle$ is without repetitions, then for some stationary subset $R^{\prime}$ of $R$ the sequence $\left\langle f_{\alpha_{\varepsilon}}(n): \varepsilon \in R^{\prime}\right\rangle$ is increasing. 
We also have

$\otimes_{2}$ for every $\beta^{\prime}<\beta^{*}$ there is $\beta^{\prime \prime} \in\left(\beta^{\prime}, \beta^{*}\right)$ such that $\left\{\alpha \in S_{\aleph_{0}}^{\lambda}\right.$ : $\left.f_{\alpha}(n(*)) \in\left[\beta^{\prime}, \beta^{\prime \prime}\right)\right\}$ is a stationary subset of $\lambda$.

[Why? Follows from $\otimes_{1}$ as $\aleph_{1}<\lambda$.]

As $\beta^{*} \in N$ we can find an increasing continuous sequence $\left\langle\beta_{\xi}: \xi<\omega_{1}\right\rangle \in$ $N$ of ordinals with limit $\beta^{*}$. So by $\otimes_{2}$ we have

$\otimes_{3}$ for every $\xi_{1}<\omega_{1}$ for some $\xi_{2} \in\left(\xi_{1}, \omega_{1}\right)$ the set $\left\{\alpha \in S_{\aleph_{0}}^{\lambda}: f_{\alpha}(n(*)) \in\right.$ $\left.\left[\beta_{\xi_{1}}, \beta_{\xi_{2}}\right)\right\}$ is stationary.

Hence for some unbounded subset $u$ of $\omega_{1}$ we have

$\otimes_{4}$ for every $\xi \in u$ the set $\left\{\alpha \in S_{\aleph_{0}}^{\lambda}: f_{\alpha}(n(*)) \in\left[\beta_{\xi}, \beta_{\xi+1}\right)\right\}$ is a stationary subset of $\lambda$.

If $2^{\aleph_{1}} \leq \mu$ then $u=\omega_{1}$, recalling we demand $\left\langle\beta_{\xi}: \xi<\omega_{1}\right\rangle \in N$.

We define $h: \lambda \rightarrow \omega_{1}$ by $h(\alpha)=\zeta$ iff we have $\zeta=\operatorname{otp}\left(\left\{f_{\beta_{\xi}}(n(*))\right.\right.$ : $\left.\xi \in u\} \cap f_{\alpha}(n(*))\right)$ and/or $\xi=0 \& f_{\alpha}(n(*)) \geq \beta^{*}$.

Clearly $h \in N$ is as required. So $\bar{S}=\bar{S}^{h}$ as required exists. But maybe $2^{\aleph_{1}}>\mu$; then after $\otimes_{3}$ we continue as follows. Let $\bar{C}=\left\langle C_{\delta}: \delta \in S_{\aleph_{1}}^{\aleph_{3}}\right\rangle$ be such that $C_{\delta}$ is a club of $\delta$ of order type $\omega_{1}$ which guesses clubs, i.e. for every club $C$ of $\aleph_{3}$ for stationarily many $\delta \in S_{\aleph_{1}}^{\aleph_{3}}$ we have $C_{\delta} \subseteq C$ (exists by [Sh:g, III]). Without loss of generality $\bar{C} \in N$.

Now let $\delta_{*} \in \operatorname{acc}\left(C^{*}\right)$ have cofinality $\aleph_{3}$. Some increasing continuous sequence $\left\langle\alpha_{\varepsilon}: \varepsilon<\aleph_{3}\right\rangle$ with $\alpha_{\varepsilon} \in \operatorname{acc}\left(C^{*}\right)$ has limit $\delta_{*}$. Now for each $\varepsilon \in S_{\aleph_{1}}^{\aleph_{3}}$ we could choose above $\delta=\alpha_{\varepsilon}$, hence for some $n_{\varepsilon}<\omega$ we have $\left(\forall \beta^{\prime}<\alpha_{\varepsilon}\right)$ $\left(\exists \beta^{\prime \prime}<\alpha_{\varepsilon}\right)\left[\beta^{\prime}<\beta^{\prime \prime} \wedge\left(\exists^{\text {stat }} \gamma \in S_{\aleph_{0}}^{\lambda}\right)\left(\beta^{\prime} \leq f_{\gamma}\left(n_{\varepsilon}\right)<\beta^{\prime \prime}\right]\right.$. So for some $n_{*}<\omega$ the set $S^{\prime}:=\left\{\varepsilon<\aleph_{3}: \operatorname{cf}(\varepsilon)=\aleph_{1}\right.$ and $\left.n_{\varepsilon}=n_{*}\right\}$ is a stationary subset of $S_{\aleph_{1}}^{\aleph_{3}}$. It follows that $\left(\forall \varepsilon<\aleph_{3}\right)\left(\exists \zeta<\aleph_{3}\right)\left[\varepsilon<\zeta \wedge\left(\exists^{\text {stat }} \gamma \in S_{\aleph_{0}}^{\lambda}\right)\left(\alpha_{\zeta} \leq f_{\gamma}\left(n_{*}\right)\right.\right.$ $\left.\left.<\alpha_{\zeta+1}\right)\right]$.

Let $\zeta_{\varepsilon}$ be the minimal $\zeta$ as required above, so $C=\left\{\xi<\aleph_{3}\right.$ : if $\varepsilon<\xi$ then $\zeta_{\varepsilon}<\xi$ and $\xi$ is a limit ordinal $\}$ is a club of $\aleph_{3}$. Hence for some $\varepsilon(*) \in S_{\aleph_{1}}^{\aleph_{3}}$ we have $C_{\varepsilon(*)} \subseteq C$. Let $u:=\left\{\beta_{\zeta}: \zeta \in C_{\varepsilon(*)}\right\}$ so clearly $\left\langle\beta_{\zeta}: \zeta \in u\right\rangle$ belongs to $N$, and define $h$ as above for $u=\omega_{1} \cdot \mathbf{m}_{1.5}$

1.7. REmarK. Why can't we, in the proof of 1.5 , after $\otimes_{3}$, instead of assuming $2^{\aleph_{1}} \leq \mu$, use "as $\left.N \prec\left(\mathscr{H}\left(2^{\lambda}\right)^{+}\right), \in,<^{*}\right)$ without loss of generality $u=\omega_{1} " ?$

The set $u$ chosen above depends on $\delta$, so if $2^{\aleph_{1}} \leq \mu$ still $u \in N$, but otherwise the "without loss of generality $u \in N$ " does not seem to be justified.

Continuation of the proof of 1.1. Let $S:=\bigcup\left\{S_{\varepsilon}: \varepsilon<\omega_{1}\right\}$. For $\varepsilon<\omega_{1}$ and $\delta \in S_{\varepsilon}$ let 


$$
\begin{aligned}
& \mathscr{A}_{\delta}^{\varepsilon}=\left\{a: a \in[\delta]^{\aleph_{0}} \text { is } M^{*} \text {-closed, } \sup (a)=\delta, \operatorname{otp}(a) \leq \varepsilon\right. \text { and } \\
& \left.\quad\left(\forall^{D_{1, \varepsilon}} n\right)\left(a \cap \lambda_{n} \subseteq f_{\delta}(n)\right) \text { and }\left(\forall^{D_{2, \varepsilon}} n\right)\left(a \cap \lambda_{n} \nsubseteq f_{\delta}(n)\right)\right\}, \\
& \mathscr{A}^{\varepsilon}=\bigcup\left\{\mathscr{A}_{\delta}^{\varepsilon}: \delta \in S_{\varepsilon}\right\}, \quad \mathscr{A}=\bigcup\left\{\mathscr{A}^{\varepsilon}: \varepsilon<\omega_{1}\right\} .
\end{aligned}
$$

So

$$
\mathscr{A} \subseteq[\lambda]^{\aleph_{0}}
$$

As the case $\mu_{*}=\aleph_{2}$ was the original question and its proof is simpler, we first prove it.

1.8. SubClaim. $\mathscr{A}$ does not reflect in any $A \in[\lambda]^{\aleph_{1}}$.

Proof. So assume $A \in[\lambda]^{\aleph_{1}}$, let $\left\langle a_{i}: i<\omega_{1}\right\rangle$ be an increasing continuous sequence of countable subsets of $A$ with union $A$, and let $R=\left\{i<\omega_{1}\right.$ : $\left.a_{i} \in \mathscr{A}\right\}$, and assume toward a contradiction that $R$ is a stationary subset of $\omega_{1}$. As every $a \in \mathscr{A}$ is $M^{*}$-closed, necessarily $A$ is $M^{*}$-closed and so without loss of generality each $a_{i}$ is $M^{*}$-closed.

For each $i \in R$, as $a_{i} \in \mathscr{A}$, by the definition of $\mathscr{A}$ we can find $\varepsilon_{i}<\omega_{1}$ and $\delta_{i} \in S_{\varepsilon_{i}}$ such that $a_{i} \in \mathscr{A}_{\delta_{i}}^{\varepsilon_{i}}$, hence by the definition of $\mathscr{A}_{\delta_{i}}^{\varepsilon_{i}}$ we have $\operatorname{otp}\left(a_{i}\right) \leq \varepsilon_{i}$. But as $A=\bigcup\left\{a_{i}: i<\omega_{1}\right\}$ with $a_{i}$ countable increasing with $i$ and $|A|=\aleph_{1}$, clearly for some club $E$ of $\omega_{1}$ the sequence $\left\langle\operatorname{otp}\left(a_{i}\right): i \in E\right\rangle$ is strictly increasing, hence $i \in E \Rightarrow \operatorname{otp}(i \cap E) \leq \operatorname{otp}\left(a_{i}\right)$, so without loss of generality $i \in E \Rightarrow i \leq \operatorname{otp}\left(a_{i}\right)$ and $i<j \in E \Rightarrow \varepsilon_{i}<j \leq \operatorname{otp}\left(a_{j}\right)$.

Now $j \in E \cap R \Rightarrow j \leq \operatorname{otp}\left(a_{j}\right) \leq \varepsilon_{j}$, so $\left\langle\varepsilon_{i}: i \in E \cap R\right\rangle$ is strictly increasing; but $\left\langle S_{\varepsilon}: \varepsilon<\omega_{1}\right\rangle$ are pairwise disjoint and $\delta_{i} \in S_{\varepsilon_{i}}$ so $\left\langle\delta_{i}: i \in\right.$ $E \cap R\rangle$ is without repetitions; but $\delta_{i}=\sup \left(a_{i}\right)$ and for $i<j$ from $R \cap E$ we have $a_{i} \subseteq a_{j}$, which implies that $\delta_{i}=\sup \left(a_{i}\right) \leq \sup \left(a_{j}\right)=\delta_{j}$, so necessarily $\left\langle\delta_{i}: i \in R \cap E\right\rangle$ is strictly increasing.

As $\sup \left(a_{i}\right)=\delta_{i}$ for $i \in R \cap E$, clearly $\sup (A)=\bigcup\left\{\delta_{i}: i \in E \cap R\right\}$. Let $\beta_{i}=\operatorname{Min}\left(A \backslash \delta_{i}\right)$ for $i<\omega_{1}$; it is well defined as $\left\langle\delta_{j}: j \in R \cap E\right\rangle$ is strictly increasing. Thinning out $E$, without loss of generality we have

$\circledast_{1} i<j \in E \cap R \Rightarrow \beta_{i}<\delta_{j} \& \beta_{i} \in a_{j}$.

Note that, by the choice of $M^{*}$,

$\circledast_{2}(i \in E \cap R \wedge i<j \in E \cap R) \Rightarrow \beta_{i} \in a_{j} \Rightarrow \bigwedge_{n}\left(f_{\beta_{i}}(n) \in a_{j}\right)$ $\Rightarrow \bigwedge_{n}\left(f_{\beta_{i}}(n)+1 \in a_{j}\right)$.

As $\left\langle\delta_{i}: i \in E \cap R\right\rangle$ is (strictly) increasing continuous and $R \cap E$ is a stationary subset of $\omega_{1}$, clearly by clause (D) of the assumption of 1.1 we can find a stationary $R_{1} \subseteq E \cap R$ and $n(*)$ such that $\left(i \in R_{1} \wedge j \in R_{1} \wedge i<j \wedge n(*) \leq\right.$ $n<\omega) \Rightarrow f_{\delta_{i}}(n)<f_{\delta_{j}}(n)$.

Now if $i \in R_{1}$, let $\mathbf{j}(i):=\operatorname{Min}\left(R_{1} \backslash(i+1)\right)$, so $f_{\delta_{i}} \leq_{J_{\omega}^{\mathrm{bd}}} f_{\beta_{i}}<_{J_{\omega}^{\mathrm{bd}}} f_{\delta_{\mathbf{j}(i)}}$, so for some $m_{i}<\omega$ we have $n \in\left[m_{i}, \omega\right) \Rightarrow f_{\delta_{i}}(n) \leq f_{\beta_{i}}(n)<f_{\delta_{\mathbf{j}(i)}}(n)$. Clearly for some stationary $R_{2} \subseteq R_{1}$ we have $i, j \in R_{2} \Rightarrow m_{i}=m_{j}=m(*)$, so 
possibly increasing $n(*)$ without loss of generality $n(*) \geq m(*)$; so we have (with $\mathrm{Ch}_{a} \in \prod_{n<\omega} \lambda_{n}$ defined by $\mathrm{Ch}_{a}(n)=\sup \left(a \cap \lambda_{n}\right)$ for any $\left.a \in[\mu]^{<\lambda_{0}}\right)$ :

$\circledast_{3}$ for $i<j$ from $R_{2}$ we have $\mathbf{j}(i) \leq j$ and

$(\alpha) f_{\delta_{i}}\left\lceil[n(*), \omega) \leq f_{\beta_{i}}\lceil[n(*), \omega)\right.$,

( $\beta) f_{\beta_{i}}\left\lceil[n(*), \omega)<f_{\delta_{\mathrm{j}(i)}}\left\lceil[n(*), \omega) \leq f_{\delta_{j}}\lceil[n(*), \omega)\right.\right.$,

$(\gamma) f_{\beta_{i}}\left\lceil[n(*), \omega)<\mathrm{Ch}_{a_{j}}\left\lceil[n(*), \omega)\right.\right.$, by $\circledast_{2}$.

Now by the definition of $\mathscr{A}_{\delta_{i}}^{\varepsilon_{i}}$, as $a_{i} \in \mathscr{A}_{\delta_{i}}^{\varepsilon_{i}} \subseteq \mathscr{A}^{\varepsilon_{i}}$ we have

$\circledast_{4}$ if $i \in R_{2}$ then

( $\alpha) \mathrm{Ch}_{a_{i}} \leq{ }_{D_{1, \varepsilon_{i}}} f_{\delta_{i}}$

(ß) $f_{\delta_{i}}<{ }_{D_{2, \varepsilon_{i}}} \mathrm{Ch}_{a_{i}}$.

Let $f^{*} \in \prod_{n<\omega} \lambda_{n}$ be $f^{*}(n)=\bigcup\left\{f_{\beta_{i}}(n): i \in R_{2}\right\}$ if $n \geq n(*)$ and zero otherwise. As $f_{\beta_{i}}(n) \in a_{j(i)}$ for $i \in R_{2}$, by $\circledast_{3}(\gamma)$ clearly $n \geq n(*) \Rightarrow$ $f^{*}(n) \leq \sup \left\{\mathrm{Ch}_{a_{i}}(n): i \in R_{2}\right\}=\sup \left(A \cap \lambda_{n}\right)=\mathrm{Ch}_{A}(n)$ and by $\circledast_{3}(\beta)$ we have $n \geq n(*) \Rightarrow \operatorname{cf}\left(f^{*}(n)\right)=\aleph_{1}$. Let $B_{1}:=\{n<\omega: n \geq n(*)$ and $\left.f^{*}(n)=\sup \left(A \cap \lambda_{n}\right)\right\}$ and $B_{2}:=[n(*), \omega) \backslash B_{1}$. As $\alpha \in A \Rightarrow \alpha+1 \in A$ we have $n \in B_{1} \Rightarrow A \cap \lambda_{n} \subseteq f^{*}(n)=\sup \left(A \cap \lambda_{n}\right)$. Also by a previous sentence $f^{*}\left\lceil[n(*), \omega) \leq \mathrm{Ch}_{A}\left\lceil[n(*), \omega)\right.\right.$, so clearly $n \in B_{2} \Rightarrow A \cap \lambda_{n} \nsubseteq f^{*}(n)$. As $\left\langle a_{i}: i \in R_{2}\right\rangle$ is increasing with union $A$, clearly there is $i(*) \in R_{2}$ such that $n \in B_{2} \Rightarrow a_{i(*)} \cap \lambda_{n} \nsubseteq f^{*}(n)$, so as $i \in R_{2} \& \alpha \in a_{i} \Rightarrow \alpha+1 \in a_{i}$ we have $i(*) \leq i \in R_{2} \Rightarrow \mathrm{Ch}_{a_{i}}\left\lceil B_{2}>f_{\delta_{i}}\left\lceil B_{2}\right.\right.$, hence by clause $\circledast_{4}(\alpha)$ we have $i \in R_{2} \backslash i(*) \Rightarrow B_{2}=\emptyset \bmod D_{1, \varepsilon_{i}} \Rightarrow B_{1} \in D_{1, \varepsilon_{i}}$. Also by $\circledast_{3}$ and the choice of $f^{*}$ and $B_{1}$, for each $n \in B_{1}$ for some club $E_{n}$ of $\omega_{1}$ we have $i \in E_{n} \cap R_{2} \Rightarrow$ $\sup \left(a_{i} \cap \lambda_{n}\right)=\sup \left\{f_{\beta_{j}}(n): j \in R_{2} \cap i\right\}=\sup \left\{f_{\delta_{j}}(n): j \in R_{2} \cap i\right\} \subseteq f_{\delta_{i}}(n)$, hence $R_{3}=R_{2} \cap \bigcap\left\{E_{n} \backslash i(*): n \in B_{1}\right\}$ is a stationary subset of $\omega_{1}$. So $n \in$ $B_{1} \& i \in R_{3} \Rightarrow a_{i} \cap \lambda_{n} \subseteq f_{\delta_{i}}(n)$, hence $i \in R_{3} \Rightarrow \mathrm{Ch}_{a_{i}}\left|B_{1} \leq f_{\delta_{i}}\right| B_{1}$, hence by $\circledast_{4}(\beta)$ we have $i \in R_{3} \Rightarrow B_{1}=\emptyset \bmod D_{2, \varepsilon_{i}}$, hence $i \in R_{3} \Rightarrow B_{2} \in D_{2, \varepsilon_{i}}$.

By the choice of $\left\langle\left(D_{1, i}, D_{2, i}\right): i<\omega_{1}\right\rangle$ in 1.5 , as $B_{1} \cup B_{2}$ is a cofinite subset of $\omega$, and $B_{1} \cap B_{2}=\emptyset$ (by the choice of $B_{1}, B_{2}$, clearly) and $R_{3} \subseteq \omega_{1}$ is stationary, we get a contradiction (see $(*)_{3}$ (iii) of 1.5$)$. $\mathbf{m}_{1.8}$

\subsection{Subclaim. $\mathscr{A}$ is a stationary subset of $[\lambda]^{\aleph_{0}}$.}

Remark. See [RuSh 117], [Sh:f, XI, 3.5, p. 546], [Sh:f, XV, 2.6].

We give a proof relying only on [Sh:f, XI, 3.5, p. 546]. In fact, also if we are interested in $\mathrm{Ch}_{N}=\left\langle\sup (\theta \cap N): \aleph_{0}<\theta \in N \cap \operatorname{Reg}\right\rangle, N \prec(\mathscr{H}(\chi), \in)$, we have full control, e.g., if $\bar{S}=\left\langle S_{\theta}: \aleph_{1} \leq \theta \in \operatorname{Reg} \cap \chi\right\rangle, S_{\theta} \subseteq S_{\aleph_{0}}^{\theta}$ stationary, we can demand $\aleph_{1} \leq \theta=\operatorname{cf}(\theta) \wedge \theta \in N \Rightarrow \mathrm{Ch}_{N}(\theta) \in S_{\theta}$ and control the order of $f_{\sup (N \cap \lambda)}^{\mathfrak{a}, \lambda}$ and $\mathrm{Ch}_{N}\lceil\mathfrak{a}$.

Proof of Subclaim 1.9. Let $M^{* *}$ be an expansion of $M^{*}$ by countably many functions; without loss of generality $M^{* *}$ has Skolem functions. 
Recall that $S^{*} \subseteq S_{\aleph_{1}}^{\lambda}$ is from 1.5 , so it belongs to $\check{I}[\lambda]$, and let $\bar{a}=\left\langle a_{\alpha}\right.$ : $\alpha<\lambda\rangle$ witness it (see $0.6,0.7$ ), so otp $\left(a_{\alpha}\right) \leq \omega_{1}$ and $\beta \in a_{\alpha} \Rightarrow a_{\beta}=\beta \cap a_{\alpha}$, and omitting a non-stationary subset of $S^{*}$ we have $\delta \in S^{*} \Rightarrow \operatorname{otp}\left(a_{\delta}\right)=$ $\omega_{1} \& \delta=\sup \left(a_{\delta}\right)$.

Let

$T^{*}=\{\eta: \eta$ is a finite sequence of ordinals,

$$
\left.\eta(2 n)<\lambda \text { and } \eta(2 n+1)<\lambda_{n}\right\} .
$$

Let $\lambda_{\eta}=\lambda$ if $\lg (\eta)$ is even and $\lambda_{\eta}=\lambda_{n}$ if $\lg (\eta)=2 n+1$, and let $\mathbf{I}_{\eta}$ be the non-stationary ideal on $\lambda_{\eta}$ for $\eta \in T^{*}$, so $\left(T^{*}, \overline{\mathbf{I}}\right)$ is well defined where $\overline{\mathbf{I}}:=\left\langle\mathbf{I}_{\eta}: \eta \in T^{*}\right\rangle$.

For $\eta \in T^{*}$, let $M_{\eta}$ be the $M^{* *}$-closure of $\{\eta(l): l<\lg (\eta)\}$ so each $M_{\eta}$ is countable and $\eta \triangleleft \nu \in T^{*} \Rightarrow M_{\eta} \subseteq M_{\nu}$, and for $\eta \in \lim \left(T^{*}\right)=\left\{\eta \in{ }^{\omega} \lambda\right.$ : $\eta\left\lceil n \in T^{*}\right.$ for every $\left.n<\omega\right\}$ let $M_{\eta}=\bigcup\left\{M_{\eta \mid n}: n<\omega\right\}$. It is enough to prove that $M_{\eta} \in \mathscr{A}$ for some $\eta \in \lim \left(T^{*}\right)$, more exactly $\left|M_{\eta}\right| \in \mathscr{A}$; recall $M_{\eta} \subseteq$ $M^{* *} \Leftrightarrow M_{\eta} \prec M^{* *}$ as $M^{* *}$ has Skolem functions. Let $\bar{M}=\left\langle M_{\eta}: \eta \in T^{*}\right\rangle$. Then we can find a subtree $T \subseteq T^{*}$ such that

$\nabla\left(T^{*}, \overline{\mathbf{I}}\right) \leq(T, \overline{\mathbf{I}})$ and for some $\varepsilon^{*}<\omega_{1}$ we have $\eta \in \lim (T) \Rightarrow$ $\operatorname{otp}\left(M_{\eta}\right)=\varepsilon^{*}$ (recalling $\left(T^{*}, \overline{\mathbf{I}}\right) \leq(T, \overline{\mathbf{I}})$ means $T \subseteq T^{*},\left(\forall \eta \in T^{*}\right)$ $(\forall l<\lg (\eta))\left(\eta\left\lceil l \in T^{*}\right),<>\in T^{*}\right.$ and $(\forall \eta \in T)\left(\left\{\alpha<\lambda_{\eta}: \eta^{\wedge}\langle\alpha\rangle \in\right.\right.$ $\left.T^{*}\right\} \neq \emptyset \bmod \mathbf{I}_{\eta}$, i.e. is stationary)).

Why? As $\lim \left(T^{*}\right)=\bigcup\left\{\mathbf{B}_{\varepsilon}: \varepsilon<\omega_{1}\right\}$ (see $\otimes_{4}$ below), and by $\otimes_{1}$ below,

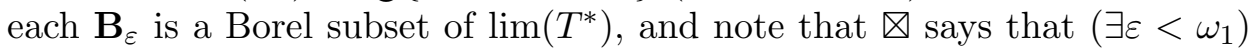
$(\exists T)\left[\left(T^{*}, \overline{\mathbf{I}}\right) \leq(T, \overline{\mathbf{I}}) \cap \lim (T) \subseteq \mathbf{B}_{\varepsilon}\right)$. For the existence of such $\varepsilon$ see, e.g., [Sh:f, XI, 3.5, p. 546]; the reader may ask to justify the sets being Borel, so let $u_{\eta}$ be the universe of $M_{\eta}$, a countable set of ordinals.

So we use

$\bigotimes_{1}$ for any $\varepsilon<\omega_{1}$ the set $\mathbf{B}_{\varepsilon}=\left\{\eta \in \lim (T): \operatorname{otp}\left(u_{\eta}\right)=\varepsilon\right\}$ is a Borel set.

[Why? Without loss of generality $u_{\eta} \neq \emptyset$ and let $\left\langle\alpha_{\eta, n}: n<\omega\right\rangle$ enumerate the members of $u_{\eta}$ and for $n_{1}, n_{2}<\omega$ and $m_{1}, m_{2}<\omega$ let $\mathbf{B}_{n_{1}, n_{2}, m_{1}, m_{2}}:=$ $\left\{\eta \in \lim \left(T^{*}\right): \alpha_{\eta\left\lceil n_{1}, m_{1}\right.}<\alpha_{\eta\left\lceil n_{2}, m_{2}\right.}\right\}$.

Clearly

$\bigotimes_{2} \quad \mathbf{B}_{n_{1}, n_{2}, m_{1}, m_{2}}$ is an open subset of $\lim \left(T^{*}\right)$,

$\otimes_{3}$ there is an $\mathbb{L}_{\omega_{1}, \omega}$ sentence $\psi_{\varepsilon}$ in the vocabulary consisting of $\left\{p_{n_{1}, n_{2}, m_{1}, m_{2}, l}: n_{1}, n_{2}, m_{1}, m_{2}<\omega\right\}$ such that: the $p$ 's are propositional variables (i.e. 0 -place predicates) and if $\left\langle\alpha_{n, m}: n, m<\omega\right\rangle$ is a sequence of ordinals and $p_{n_{1}, n_{2}, m_{1}, m_{2}}$ is assigned the truth value of $\alpha_{n_{1}, m_{1}}<\alpha_{n_{2}, m_{2}}$ then $\gamma=\operatorname{otp}\left\{\alpha_{n, m}: n, m<\omega\right\}$ iff $\psi_{\varepsilon}$ is assigned the truth value true.] 
$\bigotimes_{4} \lim \left(T^{*}\right)=\bigcup\left\{\mathbf{B}_{\varepsilon}: \varepsilon<\omega_{1}\right\}$.

[Why? As otp $\left(M_{\eta} \cap \lambda\right)<\left\|M_{\eta}\right\|^{+}=\aleph_{1}$. Together $\otimes$ should be clear.]

Note that for every $\eta \in T^{*}$ of length $2 n+2$ we have $\eta \unlhd \nu \in T^{*} \Rightarrow \mathbf{I}_{\nu}$ is $\lambda_{n}^{+}$-complete. As we can shrink $T$ further by [Sh:f, XI, 3.5, p. 346], without loss of generality

$\otimes$ for every $n<\omega$ and $\eta \in T \cap{ }^{2 n+2} \lambda$ for some $\alpha=\alpha_{\eta}<\lambda_{n}$ we have: if $\eta \triangleleft \nu \in \lim (T)$ then $\alpha_{\eta}=\sup \left(\lambda_{n} \cap M_{\nu}\right)$.

[Why? As above applied to each $T^{\prime}=\left\{\varrho \in{ }^{\omega>\lambda} \lambda: \eta^{\wedge} \varrho \in T\right\}$.]

Let $\chi=\left(2^{\lambda}\right)^{+}$and let $N_{\alpha}^{*} \prec \mathfrak{B}=\left(\mathscr{H}(\chi), \in,<_{\chi}^{*}\right)$ for $\alpha<\lambda$ be increasing continuous, $\left\|N_{\alpha}^{*}\right\|=\mu, \alpha \subseteq N_{\alpha}^{*},\left\langle N_{\beta}^{*}: \beta \leq \alpha\right\rangle \in N_{\alpha+1}^{*}$ and $(T, \overline{\mathbf{I}}, \bar{M}, \bar{a}, \bar{f}, \bar{\lambda}, \mu) \in N_{\alpha}^{*}$ (clearly possible) and $E=\left\{\delta<\lambda: N_{\delta}^{*} \cap \lambda=\delta\right\}$ is a club of $\lambda$, hence we can find $\delta(*) \in S^{*} \cap E$, so $a_{\delta(*)}$ is well defined. Let $\bar{N}^{*}=\left\langle N_{\alpha}^{*}: \alpha<\lambda\right\rangle$. Let $C_{\delta(*)}$ be the closure of $a_{\delta(*)}$ as a subset of $\delta(*)$ in the order topology and let $\left\langle\alpha_{\varepsilon}: \varepsilon<\omega_{1}\right\rangle$ list $C_{\delta(*)}$ in increasing order, so it is increasing continuous.

We define $N_{\varepsilon}$ by induction on $\varepsilon<\omega_{1}$ by:

$(*)_{0} N_{\varepsilon}$ is the Skolem hull in $\mathfrak{B}$ of

$$
\left\{\alpha_{\zeta}: \zeta<\varepsilon\right\} \cup\left\{\left\langle N_{\xi}: \xi<\zeta\right\rangle, \bar{N}^{*} \zeta \zeta: \zeta<\varepsilon\right\} \cup\{(T, \overline{\mathbf{I}}, \bar{M}, \bar{a}, \bar{f}, \bar{\lambda}, \mu)\} .
$$

Let

$(*)_{1} g_{\varepsilon} \in \prod_{n<\omega} \lambda_{n}$ be defined by $g_{\varepsilon}(n)=\sup \left(N_{\varepsilon} \cap \lambda_{n}\right)$.

Clearly

$(*)_{2} \quad$ (a) $\left\langle N_{\zeta}: \zeta \leq \varepsilon\right\rangle \in N_{\delta(*)}^{*}$ and even $\in N_{\xi}$ for every $\xi \in\left[\varepsilon+1, \omega_{1}\right)$,

(b) $C_{\delta(*)} \cap\left(\alpha_{\varepsilon}+1\right)$ and $a_{\alpha_{\varepsilon}}$ belong to $N_{\xi}$ for $\xi \in\left[\varepsilon+1, \omega_{1}\right)$ for $\varepsilon$ non-limit.

[Why? For clause (a), $\left\langle N_{\zeta}: \zeta \leq \varepsilon\right\rangle$ appears in the set whose Skolem hull is $N_{\xi}$. For clause (b) because $\bar{a} \in N_{\delta(*)}^{*}$ and $\alpha \in a_{\delta(*)} \Rightarrow a_{\alpha}=a_{\delta(*)} \cap \alpha$ and $C_{\delta(*)} \cap\left(\alpha_{\varepsilon}+1\right)=$ the closure of $a_{\alpha_{\varepsilon+1}} \cap\left(\alpha_{\varepsilon}+1\right)$.]

Let $e=\left\{\varepsilon<\omega_{1}: \varepsilon\right.$ is a limit ordinal and $\left.N_{\varepsilon} \cap \omega_{1}=\varepsilon\right\}$. So

$(*)_{3}$ (a) $e$ is a club of $\omega_{1}$,

(b) if $\varepsilon \in e$ then $\sup \left(N_{\varepsilon} \cap \lambda\right)=\alpha_{\varepsilon}=N_{\alpha_{\varepsilon}}^{*} \cap \lambda, N_{\varepsilon} \subseteq N_{\alpha_{\varepsilon}}^{*}$ and $\varepsilon<\zeta<\omega_{1} \Rightarrow N_{\varepsilon} \in N_{\zeta}$,

hence

$(*)_{4}$ if $\varepsilon+2<\zeta \in e$ then $g_{\varepsilon}, g_{\varepsilon+1} \in N_{\varepsilon+2} \prec N_{\zeta}$.

Now $\bar{f}$ is increasing and cofinal in $\prod_{n<\omega} \lambda_{n}$, hence

$(*)_{5}$ if $\varepsilon<\zeta \in e$ then $g_{\varepsilon}<_{J_{\omega}^{\text {bd }}} f_{\alpha_{\zeta}}$ and $f_{\alpha_{\varepsilon}}<_{J_{\omega}}$ bd $g_{\zeta}$. 
Also clearly

$(*)_{6}$ if $\varepsilon<\zeta \in e$ then $g_{\varepsilon}<g_{\zeta}$.

For $n<\omega$ and $\varepsilon<\omega_{1}$ let $N_{\varepsilon, n+1}$ be the Skolem hull inside $\mathfrak{B}$ of $N_{\varepsilon} \cup \lambda_{n}$ and let $N_{\varepsilon, 0}=N_{\varepsilon}$. Clearly

$$
(*)_{7} \text { if } n \leq m<\omega \text { and } \varepsilon<\omega_{1} \text { then } g_{\varepsilon}(m)=\sup \left(N_{\varepsilon, n} \cap \lambda_{m}\right) .
$$

Recall that $\varepsilon^{*}$ is the order type of $M_{\eta} \cap \lambda$ for every $\eta \in \lim (T)$. Choose $\varepsilon \in \operatorname{acc}(e)$ such that $\varepsilon>\varepsilon^{*}$ and $\alpha_{\varepsilon} \in S_{\zeta}$ for some $\zeta \in\left[\varepsilon, \omega_{1}\right)$ (possible by Subclaim 1.5, particularly clause $\left.(*)_{2}(\mathrm{ii})\right)$ and choose $\varepsilon_{k} \in e \cap \varepsilon$ for $k<\omega$ such that $\varepsilon_{k}<\varepsilon_{k+1}<\varepsilon=\bigcup\left\{\varepsilon_{l}: l<\omega\right\}$. We also choose $n_{k}$ by induction on $k<\omega$ such that

$$
\begin{aligned}
& (*)_{8} \text { (a) } n_{l}<n_{k}<\omega \text { for } l<k, \\
& \text { (b) } \left.g_{\varepsilon_{k+1}} \uparrow\left[n_{k}, \omega\right)<f_{\alpha_{\varepsilon}}\right\rceil\left[n_{k}, \omega\right) .
\end{aligned}
$$

[Why is this choice possible? By $(*)_{5}$.]

We stipulate $n_{-1}=0$.

Let $B_{1} \in D_{1, \zeta}$ be such that $B_{2}=\omega \backslash B_{1} \in D_{2, \zeta}$ (exists by clause $(*)_{3}$ (iv) of Subclaim 1.5).

Now we choose $\eta_{n}$ by induction on $n<\omega$ such that

$\square$ (a) $\eta_{n} \in T$ and $\lg \left(\eta_{n}\right)=n$,

(b) $m<n \Rightarrow \eta_{m} \triangleleft \eta_{n}$,

(c) if $n \in\left[n_{k-1}, n_{k}\right)$ then $\eta_{2 n}, \eta_{2 n+1} \in N_{\varepsilon_{k}, n}$,

(d) if $n \in\left[n_{k-1}, n_{k}\right)$ then $\eta_{2 n+1}(2 n)=\operatorname{Min}\left\{\alpha<\lambda: \eta_{2 n}{ }^{\wedge}\langle\alpha\rangle \in T\right.$ and $\alpha \geq \alpha_{\varepsilon_{k-1}}$ if $\left.k>0\right\}$

(e) if $n \in\left[n_{k-1}, n_{k}\right)$ and $n \in B_{1}$ then $\eta_{2 n+2}(2 n+1)=\operatorname{Min}\left\{\alpha<\lambda_{n}\right.$ : $\left.\eta_{2 n+1} \hat{\wedge}\langle\alpha\rangle \in T\right\}$,

(f) if $n \in\left[n_{k-1}, n_{k}\right)$ and $n \in B_{2}$ then $\eta_{2 n+2}(2 n+1)=\operatorname{Min}\left\{\alpha<\lambda_{n}\right.$ : $\eta_{2 n+1} \hat{\wedge}\langle\alpha\rangle \in T$ and $\left.\alpha>f_{\alpha_{\varepsilon}}(n)\right\}$.

No problem to carry the induction.

[Clearly if $\eta_{n}$ is well defined then $\eta_{n+1}(n)$ is well defined (by clause (d), (e) or (f) according to the case); hence $\eta_{n+1} \in T \cap^{n+1} \lambda$ is well defined by why clause (c) holds, i.e. assume $n \in\left[n_{k-1}, n_{k}\right)$; why $\eta_{2 n}, \eta_{2 n+1} \in N_{\varepsilon_{k, n}}$ ?

CASE 1: If $n=0$, then $\eta_{2 n}=<>\in N_{\varepsilon_{k}, n}$ trivially.

CASE 2: $\eta_{2 n}$ is O.K., hence $\in N_{\varepsilon_{k, n}}$ and we show $\eta_{2 n+1} \in N_{\varepsilon_{k, n}}$. [Why? Because $N_{\varepsilon_{k}, n} \prec \mathfrak{B}$, if $k=0$ as $\eta_{2 n+2}(2)$ is defined from $\eta_{2 n}$ and $T$, both of which belong to $N_{\varepsilon_{k}, n}$. If $k>0$ we have to check that also $\alpha_{\varepsilon_{k-1}} \in$ $N_{\varepsilon_{k}, n}$, which holds by $(*)_{0}$.]

CASE 3: $\eta_{2 n+1}$ is O.K. so $\in N_{\varepsilon_{k}, n}$ and we have to show $\eta_{2 n+2} \in N_{\varepsilon_{k}, n+1}$. (As $\eta_{2 n+2}(n)<\lambda_{n} \subseteq N_{\varepsilon_{k}, n+1}$ this should be clear.)] 
Let $\eta=\bigcup\left\{\eta_{n}: n<\omega\right\}$. Clearly $\eta \in \lim (T)$, hence $u:=\left|M_{\eta}\right| \in[\lambda]^{\aleph_{0}}$ and $M_{\eta} \subseteq M^{* *}$, hence it is enough to prove that $u \in \mathscr{A}$.

Now

$\circledast_{1} \sup (u) \leq \alpha_{\varepsilon}$.

[Why? As $\eta_{n}$ belongs to the Skolem hull of $N_{\varepsilon} \cup \mu \subseteq N_{\alpha_{\varepsilon}}^{*}$, hence $M_{\eta_{n}} \subseteq N_{\varepsilon} \subseteq N_{\alpha_{\varepsilon}}^{*}$, and $N_{\alpha_{\varepsilon}}^{*} \cap \lambda=\alpha_{\varepsilon}$ as $\alpha_{\varepsilon} \in E$.]

$\circledast_{2} \sup (u) \geq \alpha_{\varepsilon_{n}}$ for every $n<\omega$.

[By clause (d) of $\square$.]

$\circledast_{3} \sup (u)=\alpha_{\varepsilon}$.

$\left[\right.$ Why? By $\circledast_{1}+\circledast_{2}$.]

$\circledast_{4} \alpha_{\varepsilon} \in S_{\zeta}$ and $\zeta \geq \varepsilon>\varepsilon^{*}=\operatorname{otp}(u)$.

[Why? By the choice of $\varepsilon$.]

$\circledast_{5}$ If $n \geq n_{0}, n>0$ and $n \in B_{1}$ then $u \cap \lambda_{n} \subseteq f_{\alpha_{\varepsilon}}(n)$.

[Why? By the choice of $\eta_{2 n+2}(2 n+1)$, i.e., let $k$ be such that $n \in$ $\left[n_{k-1}, n_{k}\right)$, so $\eta_{2 n+1} \in N_{\varepsilon_{k}, n}$ by clause (c), and by clause (e) of $\square$ we have $\eta_{2 n+2}(2 n+1) \in \lambda_{n} \cap N_{\varepsilon_{k}, n}$, hence by $\otimes$ above, as $\eta \in \lim (T)$ we have $\alpha_{\eta \uparrow(2 n+2)}=\alpha_{\eta_{2 n+2}}=\sup \left(u \cap \lambda_{n}\right)$, and as $\bar{M} \in N_{\varepsilon_{k}, n}$ we have $\alpha_{\eta_{2 n+2}} \in N_{\varepsilon_{k}, n}$, so $\sup \left(u \cap \lambda_{n}\right)=\alpha_{\eta_{2 n+2}}<\sup \left(N_{\varepsilon_{k}, n} \cap \lambda_{n}\right)$ but the latter is equal to $\sup \left(N_{\varepsilon_{k}} \cap \lambda_{n}\right)$ by $(*)_{7}$, which is equal to $g_{\varepsilon_{k}}(n)$, which is $<f_{\alpha_{\varepsilon}}(n)$ by $(*)_{8}$, as required.]

$\circledast_{6}$ If $n \geq n_{1}$ and $n \in B_{2}$ then $u \cap \lambda_{n} \nsubseteq f_{\alpha_{\varepsilon}}(n)$.

[Why? By the choice of $\eta_{2 n+2}(2 n+1)$.]

So we are done. 1.9

This (i.e., $1.8+1.9$ ) is enough to prove 1.1 in the case $\mu_{*}=\aleph_{2}$. In general we should replace 1.8 by the following claim.

1.10. Claim. The family $\mathscr{A}$ does not reflect in any uncountable $A \in$ $[\lambda]^{<\mu_{*}}$.

Proof. Assume $A$ is a counterexample. Trivially

$\circledast_{0} A$ is $M^{*}$-closed.

For $a \in \mathscr{A}$ let $(\delta(a), \varepsilon(a))$ be such that $a \in \mathscr{A}_{\delta(a)}^{\varepsilon(a)}$, hence $\delta(a)=\sup (a) \in$ $S_{\varepsilon(a)}$ and $\operatorname{otp}(a) \leq \varepsilon(a)$. Let $\mathscr{A}^{-}=\mathscr{A} \cap[A]^{\aleph_{0}}$ and $\Gamma=\left\{\delta(a): a \in \mathscr{A}^{-}\right\}$. Of course, $\Gamma \neq \emptyset$. Assume that $\delta_{n} \in \Gamma$ for $n<\omega$ so let $\delta_{n}=\delta\left(a_{n}\right)$ where $a_{n} \in \mathscr{A}$, then necessarily $\delta_{n} \in S_{\varepsilon\left(a_{n}\right)}$. As $A$ is uncountable we can find a countable $b$ such that $a_{n} \subseteq b \subseteq A$ and $\varepsilon\left(a_{n}\right)<\operatorname{otp}(b)$ for every $n<\omega$, and as $\mathscr{A}^{-} \subseteq[A]^{\aleph_{0}}$ is stationary we can find $c$ such that $b \subseteq c \in \mathscr{A}^{-}$; so $\varepsilon(c) \geq \operatorname{otp}(c) \geq \operatorname{otp}(b)>\varepsilon\left(a_{n}\right) \& \delta_{n} \in S_{\varepsilon\left(a_{n}\right)} \& \delta\left(a_{n}\right)=\delta_{n}<\sup \left(a_{n}\right) \leq$ $\sup (c)=\delta(c)$ for each $n<\omega$. So if $\delta\left(a_{n}\right)=\delta_{n}=\delta(c)$ and $n<\omega$ then necessarily $\varepsilon\left(a_{n}\right)=\varepsilon(c)$, a contradiction, so $\delta_{n} \neq \delta(c)$; hence $\delta(c)>\delta\left(a_{n}\right)$ and, of course, $\delta(c) \in \Gamma$ so $n<\omega \Rightarrow \delta_{n}<\delta(c) \in \Gamma$. As $\delta_{n}$ for $n<\omega$ 
were any members of $\Gamma$, clearly $\Gamma$ has no last element. Let $\delta^{*}=\sup (\Gamma)$. Similarly $\operatorname{cf}\left(\delta^{*}\right)=\aleph_{0}$ is impossible, so clearly $\operatorname{cf}\left(\delta^{*}\right)>\aleph_{0}$. Let $\theta=\operatorname{cf}\left(\delta^{*}\right)$ so $\theta \leq|A|<\mu_{*}$ and $\theta$ is a regular uncountable cardinal.

As $a \in \mathscr{A}_{\delta}^{\varepsilon} \Rightarrow \sup (a)=\delta$ and $\mathscr{A}^{-} \subseteq[A]^{\aleph_{0}}$ is stationary, clearly $A \subseteq \delta^{*}=$ $\sup (A)=\sup (\Gamma)$. Let $\left\langle\delta_{i}: i<\theta\right\rangle$ be increasing continuous with limit $\delta^{*}$, and if $\delta_{i} \in S_{\varepsilon}$ then we let $\varepsilon_{i}=\varepsilon$.

For $i<\theta$ let $\beta_{i}=\operatorname{Min}\left(A \backslash \delta_{i}\right)$, so $\delta_{i} \leq \beta_{i}<\delta^{*}, \beta_{i} \in A$ and $i<j<\theta \Rightarrow$ $\beta_{i} \leq \beta_{j}$. But $i<\theta \Rightarrow \beta_{i}<\delta^{*} \Rightarrow(\exists j)\left(i<j<\theta \wedge \beta_{i}<\delta_{j}\right)$ so for some club $E_{0}$ of $\theta$ we have $i<j \in E_{0} \Rightarrow \beta_{i}<\delta_{j} \leq \beta_{j}$; as we can replace $\left\langle\delta_{i}: i<\theta\right\rangle$ by $\left\langle\delta_{i}: i \in E_{0}\right\rangle$, without loss of generality $\beta_{i}<\delta_{i+1}$, hence $\left\langle\beta_{i}: i<\theta\right\rangle$ is strictly increasing.

Let $A^{0}:=\left\{\beta_{i}: i<\theta\right\}$, let $H:[A]^{\aleph_{0}} \rightarrow \theta$ be defined by $H(b)=\sup \{i+1$ : $\left.\beta_{i} \in b\right\}$ and let $J:=\left\{R \subseteq \theta\right.$ : the family $\left\{b \in \mathscr{A}^{-}: H(b) \in R\right\}=\left\{b \in \mathscr{A}^{-}\right.$: $\left.\sup \left(\left\{i<\theta: \beta_{i} \in b\right\}\right) \in R\right\}$ is not a stationary subset of $\left.[A]^{\aleph_{0}}\right\}$. Clearly

$\circledast_{1} J$ is an $\aleph_{1}$-complete ideal on $\theta$ extending the non-stationary ideal and $\theta \notin J$ by the definition of the ideal,

$\circledast_{2}$ if $B \in J^{+}$(i.e., $\left.B \in \mathscr{P}(\theta) \backslash J\right)$ then $\left\{a \in \mathscr{A}^{-}: H(a) \in B\right\}$ is a stationary subset of $[A]^{\aleph_{0}}$.

By clause (D) of the assumption of 1.1 , for some stationary $R_{1} \in J^{+}$and $n_{i}<\omega$ for $i \in R_{1}$ we have

$\circledast_{3}$ if $i<j$ are from $R_{1}$ and $n \geq n_{i}, n_{j}$ (but $\left.n<\omega\right)$ then $f_{\beta_{i}}(n)<f_{\beta_{j}}(n)$.

Recall that

$\circledast_{4} i<j \in R_{1} \Rightarrow \beta_{i}<\delta_{j}$.

Now if $i \in R_{1}$, let $\mathbf{j}(i)=\operatorname{Min}\left(R_{1} \backslash(i+1)\right)$, so $f_{\delta_{i}} \leq J_{\omega}^{\mathrm{bd}} f_{\beta_{i}}<_{J_{\omega}} f_{\delta_{\mathbf{j}(i)}}$; hence for some $m_{i}<\omega$ we have $n \in\left[m_{i}, \omega\right) \Rightarrow f_{\delta_{i}}(n) \leq f_{\beta_{i}}(n)<f_{\delta_{\mathbf{j}(i)}}(n)$. Clearly for some $n(*)$ satisfying $\lambda_{n(*)}>\theta$ and $R_{2} \subseteq R_{1}$ from $J^{+}$we have $i \in R_{2} \Rightarrow n_{i}, m_{i} \leq n(*)$, so

$\circledast_{5}$ for $i<j$ in $R_{2}$ we have

$(\alpha) f_{\delta_{i}}\left\lceil[n(*), \omega) \leq f_{\beta_{i}}[n(*), \omega)\right.$,

(ß) $f_{\beta_{i}}\left\lceil[n(*), \omega)<f_{\delta_{j}}\lceil[n(*), \omega)\right.$.

Let $f^{*} \in \prod_{n<\omega} \lambda_{n}$ be defined by $f^{*}(n)=\bigcup\left\{f_{\delta_{i}}(n): i \in R_{2}\right\}$ if $n \geq n(*)$ and zero otherwise. Clearly $f^{*}(n) \leq \sup \left(A \cap \lambda_{n}\right)$ for $n<\omega$.

Let $\mathscr{A}^{\prime}=\left\{a \in \mathscr{A}^{-}: \sup \left\{i \in R_{2}: \beta_{i} \in a\right\}=\sup \left\{i: \beta_{i} \in a\right\}, H(b) \in R_{2}\right.$ and $\left.\sup \left(A \cap \lambda_{n}\right)>f^{*}(n) \Rightarrow a \cap \lambda_{n} \nsubseteq f^{*}(n)\right\}$. As $R_{2} \in J^{+}$clearly $\mathscr{A}^{\prime}$ is a stationary subset of $[A]^{\aleph_{0}}$.

Let $R_{3}=\left\{i \in R_{2}: i=\sup \left(i \cap R_{2}\right)\right\}$ so $R_{3} \subseteq R_{2}, R_{2} \backslash R_{3}$ is a non-stationary subset of $\theta$ (hence belongs to $J$ ) and $a \in \mathscr{A}^{\prime} \Rightarrow \sup (a) \in\left\{\delta_{i}: i \in R_{3}\right\}$. Let $\mathscr{A}^{*}$ be the set of all $a \in[A]^{\aleph_{0}}$ such that 
(a) $\beta_{\min \left(R_{2}\right)} \in a$ and $a$ is $M^{*}$-closed,

(b) if $i \in R_{2} \& j=\operatorname{Min}\left(R_{2} \backslash(i+1)\right)$ then $\left[a \nsubseteq \delta_{i} \Rightarrow a \nsubseteq \delta_{j}\right]$ and $n \in[n(*), \omega) \& a \cap \lambda_{n} \nsubseteq f_{\delta_{i}}(n) \Rightarrow a \cap \lambda_{n} \backslash f_{\delta_{j}}(n) \neq \emptyset$,

(c) if $i<\theta \& n \in[n(*), \omega)$ then $(\exists \gamma)\left(\beta_{i} \leq \gamma \in a\right) \equiv$

$(\exists j)\left(i<j<\theta \& \beta_{j} \in a\right) \equiv(\exists \gamma)\left(f_{\beta_{i}}(n) \leq \gamma \in a \cap f^{*}(n)\right)$,

(d) if $A \cap \lambda_{n} \nsubseteq f^{*}(n)$ then $a \cap \lambda_{n} \nsubseteq f^{*}(n)$

but $(\forall \gamma \in a)(\gamma+1 \in a)$ hence $\left.\sup \left(a \cap \lambda_{n}\right)>f^{*}(n)\right\}$.

Clearly $\mathscr{A}^{*}$ is a club of $[A]^{\aleph_{0}}$ (recall that $A$ is $M^{*}$-closed). But if $a \in \mathscr{A}^{*} \cap \mathscr{A}^{\prime}$, then for some limit ordinal $i \in R_{3} \subseteq \theta$ we have $a \subseteq \sup (a)=\delta_{i}$ and $n \in[n(*), \omega) \Rightarrow \sup \left(a \cap f^{*}(n)\right)=\sup \left(a \cap \bigcup\left\{f_{\delta_{j}}(n): j \in R_{2}\right\}\right)$. Let

$$
\begin{aligned}
& B_{1}=\left\{n: n(*) \leq n<\omega \text { and } A \cap \lambda_{n} \subseteq f^{*}(n)=\sup \left(A \cap \lambda_{n}\right)\right\} . \\
& B_{2}=\left\{n: n(*) \leq n<\omega \text { and } f^{*}(n)<\sup \left(A \cap \lambda_{n}\right)\right\} .
\end{aligned}
$$

Clearly $B_{1}, B_{2}$ are disjoint with union $[n(*), \omega)$ recalling $\alpha \in A \Rightarrow \alpha+1 \in A$ by $\circledast 0$.

By the definition of $\mathscr{A}^{\prime}$, for every $a \in \mathscr{A}^{\prime} \cap \mathscr{A}^{*}$, we have

$$
\begin{aligned}
& \circledast_{6} n \in B_{2} \Rightarrow \mathrm{Ch}_{a}(n) \geq f^{*}(n)>f_{\delta(a)}(n), \\
& \circledast_{7} n \in B_{1} \Rightarrow \mathrm{Ch}_{a}(n)=\bigcup\left\{f_{\beta_{\varepsilon}}(n): \varepsilon \in R_{2} \cap \delta(a)\right\} \leq f_{\delta(a)}(n) .
\end{aligned}
$$

But this contradicts the observation below.

1.11. Observation. If $B \subseteq \omega$, then for some $\varepsilon<\omega_{1}$ we have: if $a \in \mathscr{A}$ is $M^{*}$-closed and $\left\{n<\omega: \sup \left(a \cap \lambda_{n}\right) \leq f_{\sup (a)}(n)\right\}=B \bmod J_{\omega}^{\mathrm{bd}}$, then $\operatorname{otp}(a)<\varepsilon$.

Proof. Read the definition of $\mathscr{A}$ (and $\left.\mathscr{A}^{\varepsilon}, \mathscr{A}_{\delta}^{\varepsilon}\right)$ and Subclaim 1.5, particularly $(*)_{3} \cdot \mathbf{\varpi}_{1.11}, \mathbf{- 1}_{1.10}, \mathbf{- 1 . 1}_{1.1}$

REMARK. Clearly 1.11 shows that we have much freedom in the choice of $\mathscr{A}_{\delta}^{\varepsilon}$ 's.

We can get somewhat more, as in [Sh:e]:

1.12. Claim. In Claim 1.1 we can add to the conclusion

$(*) \mathscr{A}$ satisfies the diamond, i.e. $\diamond \mathscr{A}$.

Proof. In 1.5 we can add

$(*)_{4}\{2 n+1: n<\omega\}=\emptyset \bmod D_{l, \varepsilon}$ for $l<2$ and $\varepsilon<\omega_{1}$.

This is easy: replace $D_{l, \varepsilon}$ by $D_{l, \varepsilon}^{\prime}=\left\{A \subseteq \omega:\{n: 2 n \in A\} \in D_{l, \varepsilon}\right\}$. We can fix a countable vocabulary $\tau$ and for $\zeta<\omega_{1}$ choose a function $F_{\zeta}$ from $\mathscr{P}(\omega)$ onto $\{N: N$ is a $\tau$-model with universe $\zeta\}$ such that $F_{\zeta}(A)=F_{\zeta}(B)$ if $A=B \bmod$ finite.

CASE 1: $\mu>2^{\aleph_{0}}$. For $a \in \mathscr{A}$ let $\delta_{a}, \varepsilon_{a}$ be such that $a \in \mathscr{A}_{\delta_{a}}^{\varepsilon_{a}}$, let $A_{a}=\left\{n: \sup \left(a \cap \lambda_{2 n+1}\right)<f_{\delta_{a}}(2 n+1)\right\}$, and let $N_{a}$ be the $\tau$-model with universe $a$ such that the one-to-one order preserving function from $\zeta$ onto $a$ 
is an isomorphism from $F_{\zeta}\left(A_{a}\right)$ onto $N$. Note that in the proof of " $\mathscr{A} \subseteq[\lambda]^{\aleph_{0}}$ is stationary", i.e. of 1.9 , given a $\tau$-model $M$ with universe $\lambda$ without loss of generality $\lambda_{0}>2^{\aleph_{0}}$ and so we can demand that the isomorphism type of $M_{\eta}$ is the same for all $\eta \in \lim (T)$ and, of course, $M \in M_{\eta}$. Hence the isomorphism type of $M\left\lceil u_{\eta}\right.$ is the same for all $\eta \in \lim (T)$ where $u_{\eta}$ is the universe of $M_{\eta}$. Now in the choice for $B_{1}$ we can add the demand that $F_{\varepsilon^{*}}\left(\left\{n: 2 n+1 \in B_{1}\right\}\right)$ is isomorphic to $M\left\lceil u_{\eta}\right.$ for every $\eta \in \lim (T)$. Now check.

CASE 2: $\mu \leq 2^{\aleph_{0}}$. Similarly let $\{2 n+1: n<\omega\}$ be the disjoint union of $\left\langle B_{n}^{*}: n<\omega\right\rangle$, with each $B_{n}^{*}$ infinite. We use $A_{a} \cap B_{n}^{*}$ to code a model with universe $\subseteq \zeta$, for some $\zeta<\omega_{1}$, by a function $\mathbf{F}_{n}$. We then let $N_{a}$ be the model with universe $a$ such that the order preserving function from $a$ onto a countable ordinal $\zeta$ is an isomorphism from $N_{a}$ onto $\bigcup\left\{\mathbf{F}_{n}\left(A_{a} \cap B_{n}^{*}\right): n<\omega\right\}$ when the union is a $\tau$-model with universe $\zeta$.

Now we cannot demand that all $M_{\eta}, \eta \in \lim (T)$, have the same isomorphism type but only the same order type. The rest should be clear. $\mathbf{m}_{1.12}$

We can also generalize

1.13. Claim. We can weaken the assumption of 1.1 as follows:

(a) $\lambda=\operatorname{cf}(\lambda)>\mu$ instead $\lambda=\mu^{+}\left(\right.$still necessarily $\left.\mu_{*} \leq \mu\right)$,

(b) replace $J_{\omega}^{\mathrm{bd}}$ by an ideal $J$ on $\omega$ containing the finite subsets, $\lambda_{n}=$ $\operatorname{cf}\left(\lambda_{n}\right)>\aleph_{1}, \mu=\lim _{J}\left\langle\lambda_{n}: n<\omega\right\rangle$ but not necessarily $n<\omega \Rightarrow$ $\lambda_{n}<\lambda_{n+1}$ and add $\mathscr{P}(\omega) / J$ is infinite (hence uncountable).

Proof. In 1.5 in $(*)_{3}$ we choose $\left\langle A_{\varepsilon}: \varepsilon<\omega_{1}\right\rangle$, a sequence of subsets of $\omega$ such that $\left\langle A_{\varepsilon} / J: \varepsilon<\omega_{1}\right\rangle$ are pairwise distinct. This implies some changes and waiving $\lambda_{n}<\lambda_{n+1}$ requires some changes in 1.9, in particular for each $n$ using $\left\langle\mathbf{B}_{\alpha}: \alpha \in S_{\aleph_{0}}^{\lambda_{n}}\right\rangle$ with $\mathbf{B}_{\delta}=\left\{\eta \in \lim \left(T^{*}\right): a \cap \lambda_{n} \subseteq \alpha\right\}$ and the partition theorem [Sh:f, XI, 3.7, p. 549]. $\mathbf{- 1}_{1.13}$

\section{References}

[FoTo] M. Foreman and S. Todorčević, A new Löwenheim-Skolem theorem, Trans. Amer. Math. Soc. 357 (2005), 1693-1715.

[RuSh 117] M. Rubin and S. Shelah, Combinatorial problems on trees: partitions, $\Delta$ systems and large free subtrees, Ann. Pure Appl. Logic 33 (1987), 43-81.

[Sh:E3] S. Shelah, On some problems in general topology, in: Set Theory (Boise, ID, 1992-1994), Contemp. Math. 192, Amer. Math. Soc., 1996, 91-101.

[Sh:E12] - Analytical Guide and Corrections to [Sh:g], math.LO/9906022.

[Sh:e] - Non-Structure Theory, Oxford Univ. Press, to appear.

[Sh:f] - Proper and Improper Forcing, Perspectives in Math. Logic, Springer, 1998.

[Sh:g] - Cardinal Arithmetic, Oxford Logic Guides 29, Oxford Univ. Press, 1994.

[Sh 410] - More on cardinal arithmetic, Arch. Math. Logic 32 (1993), 399-428. 
[Sh 420] S. Shelah, Advances in cardinal arithmetic, in: Finite and Infinite Combinatorics in Sets and Logic, N. W. Sauer et al. (eds.), Kluwer, 1993, 355-383.

[Sh 430] - Further cardinal arithmetic, Israel J. Math. 95 (1996), 61-114.

[Sh 755] -, Weak diamond, Math. Japonica 55 (2002), 531-538; math.LO/0107207.

[Sh 775] -, Middle diamond, Arch. Math. Logic 44 (2005), 527-560.

[Ve92a] B. Veličković, Forcing axioms and stationary sets, Adv. Math. 94 (1992), 256-284.

Institute of Mathematics

Mathematics Department

The Hebrew University

Rutgers University

Jerusalem, Israel

New Brunswick, NJ 08854, U.S.A.

Received 9 June 2004;

in revised form 30 September 2007 